\title{
Breast Cancer Management during the COVID 19 Pandemic: French Guidelines
}

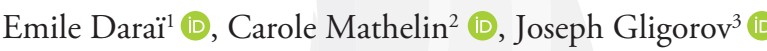 \\ ${ }^{1}$ Department of Gynaecology, Obstetrics and Human Reproductive Medicine, TENON Hospital, Paris, France \\ ${ }^{2}$ Department of Surgery, Institut de Cancérologie Strasbourg Europe (ICANS), Strasbourg, France \\ ${ }^{3}$ Executive Director of Institut Universitaire de Cancérologie AP-HP, Sorbonne Université, Paris, France
}

Cite this articles as: Daraï E, Mathelin C, Gligorov J. Breast Cancer Management during the COVID 19 Pandemic: French Guidelines. Eur J Breast Health 2020; 16(3): 160-161

At the beginning of 2020, the new coronavirus (SARS-CoV-2), responsible for severe acute respiratory syndrome, was identified as the viral agent causing pneumonia in several patients epidemiologically linked to a religious gathering in Mulhouse, a town located near Strasbourg (the town where SIS was created). Since then, the spread of this coronavirus disease (COVID-19) to many other French area was observed. In France, as of April 17 ${ }^{\text {th }}$, 2020, there were more than 109000 confirmed cases of COVID-19 and 18681 related deaths (1).

Despite extraordinary containment measures implemented from $15^{\text {th }}$ of March 2020 in France, the epidemic has spread, with clinical forms of varying severity, ranging from asymptomatic disease, minor flu-like symptoms to severe pneumopathies or multi-organ failure with a mortality rate of a few percent. Patients who are older and/or have co-morbidities (diabetes, cardiovascular disease, cancer, immunosuppression, obesity...) are the most likely to develop severe forms. The current pandemic is therefore of particular concern for cancer patients.

In France, the Nice-St Paul de Vence working group published on $9^{\text {th }}$ of April 2020 (2) a series of recommendations concerning more specifically the medical care of people with breast cancer in the context of the COVID pandemic in partnership with the Collège National des Gynécologues et Obstétriciens Français, the Société d'Imagerie de la Femme, the Société Française d'Oncologie Gynécologique, the Société Française de Sénologie et Pathologie Mammaire et the French Breast Cancer Intergroup UNICANCER. The purpose of these recommendations was double. On the one hand, oncological management must be adequate, avoiding any potential loss of opportunity with regard to breast cancer (despite the pandemic, cancer patients must have care allowing the same level of curability or life expectancy). On the other hand, cancer patients must be protected from the risk of serious or lethal infection with CoV-2-SARS.

So, many changes concerning diagnosis and treatment have therefore been decided.

The main change concerns organized screening programs, completely suspended during the pandemic period. The only suspicious lesions requiring exploration are ACR5, ACR4 and ACR3 lesions in a context of high risk. If cancer is diagnosed and requires further local assessment, this should be carried out with the aim of limiting the number of visits to imaging departments. MRI should only be used in uninfected individuals (COVID-19 negative), due to the great difficulty of adequately disinfecting the equipment.

After diagnosis, multidisciplinary meetings must be maintained but modified according to new procedures recommended by our National Institute of Cancer. With regard to the choices made during these multidisciplinary meetings, whenever an option that reduces the number of hospital visits is as effective as a treatment that requires more trips to health care facilities, it should be preferred (home administration, 3-weekly vs. weekly regimen, oral vs. intraveinous administration, hypofraction of radiotherapy, etc.). Similarly, whenever possible, tele or phone consultations should be preferred.

Concerning breast surgery, all secondary reconstruction surgeries must be postponed after the pandemic. Surgeries concerning benign lesions (atypical hyperplasia, lobular carcinoma in situ, papillomas and other benign lesions) should be deferred for 3 months. When breast surgery is to be performed, special attention is requested for extemporaneous examination, which should be performed only if absolutely necessary. Surgeries should be ambulatory as often as possible. In the case of mastectomy, immediate breast reconstruction 
with a prosthesis/expander is possible if indicated, but other more extensive techniques requiring longer surgery and hospitalization should be avoided during the pandemic. For patients with significant co-morbidities making the risk of complications high in case of COVID-19 (elderly subjects, chronic respiratory or cardiac pathology, immunosuppression...), and having a slowly evolving and hormone-dependent invasive cancer, a first hormone therapy may be proposed in order to postpone the surgical procedure.

About radiotherapy, for intraductal carcinoma, a deferral of 3 to 6 months is possible. During the pandemic, a hypofractionated regimen should be preferred. For invasive cancers that are highly hormone-dependent in postmenopausal patients, stage I or II, or in patients with significant co-morbidities exposing them to the risk of severe complications in the case of COVID-19 (elderly subjects, chronic respiratory or cardiac pathology, immunosuppression, etc.), and with an indication for radiotherapy, a deferral of 3 to 6 months is possible and a hypofractionated regimen should also be preferred. An anti-estrogens treatment can be initiated before the radiotherapy. For other invasive cancers with an indication for radiotherapy, treatment must be carried out according to the usual indications.

These recommendations are also valid for men with breast cancer.

Indications for consultation for fertility preservation should be retained.

In the case of breast cancer occurring during pregnancy, the case must be discussed during a national multidisciplinary meeting (Cancer Associated with Pregnancy, CALG Network).

Indications for oncogenetic consultations must be maintained. However, in order to reduce the number of hospital visits, the procedures for requesting a test and reporting the results are simplified, with the possibility of teleconsultation in some cases.

Travel to attend supportive care should be limited to essential care, with tele or telephone consultations being preferred. The identification of distress and the offer of psychological support are imperative during this period. Hairdressers are closed in France and the only way to obtain a hair prosthesis is online on the websites or via patient associations. The online sites of the patient's associations are recommended to patients so that they can find the additional information and social support they need during this period.

Finally, regarding clinical trials for SARS-CoV-2 infection, a history of breast cancer or current management of breast cancer should not be considered in France as an exclusion criterion for these trials alone.

It is important during the pandemic period to establish data collection regarding breast cancer patient management and the impact of treatment changes on care pathways and caregivers in order to gain valuable experience in optimizing patient management.

\section{References}

1. Available from: https://www.santepubliquefrance.fr/maladies-et-traumatismes/maladies-et-infections-respiratoires/infection-a-coronavirus/articles/ infection-au-nouveau-coronavirus-sars-cov-2-covid-19-france-et-monde.

2. Gligorov J, Bachelot T, Pierga JY, Antoine EC, Balleyguier C, Barranger E, et al. [COVID-19 and people followed for breast cancer: French guidelines for clinical practice of Nice-St Paul de Vence, in collaboration with the College Nationale des Gynecologues et Obstetriciens Francais (CNGOF), the Societe d'Imagerie de la FEMme (SIFEM), the Societe Francaise de Chirurgie Oncologique (SFCO), the Societe Francaise de Senologie et Pathologie Mammaire (SFSPM) and the French Breast Cancer IntergroupUNICANCER (UCBG)]. Bull Cancer 2020: S0007-4551(20)30159-4. DOI: 10.1016/j.bulcan.2020.03.008. (PMID: 32278467) [Crossref] 